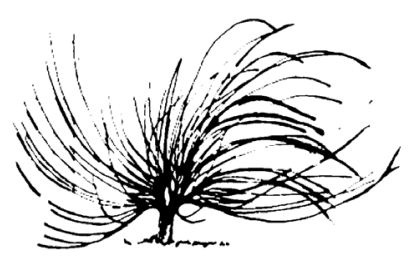

\title{
Del discurso a los hechos: Política educativa del Ministerio de Educación Pública de Costa Rica
}

\author{
Andrés Ariel Robles-Barrantes ${ }^{1}$ \\ Universidad Nacional \\ Costa Rica \\ andres.robles.barrantes@una.cr
}

\begin{abstract}
Resumen
El 13 de noviembre de 2017, el Ministerio de Educación Pública de Costa Rica (MEP), a partir de un acuerdo del Consejo Superior de Educación, emite una nueva Política Pública Educativa con el título La persona: Centro del proceso educativo y sujeto transformador de la sociedad. Enfatiza en el posicionamiento de la persona desde una perspectiva de valoración de toda su diversidad y la urgencia de que los espacios educativos cuenten con una mayor justicia y equidad. Sin embargo, es posible, por medio de un análisis discursivo crítico, descubrir la realidad en la que se encuentra el modelo educativo costarricense. Analizando los hechos vivenciales y las decisiones ejecutivas, se pueden vislumbrar los verdaderos propósitos detrás de los discursos. Una nueva política educativa no necesariamente corresponde a un cambio de paradigma. Por el contrario, a partir de los elementos teóricos, es posible contemplar, incluso, influencias de corte neoliberal que restringen los verdaderos principios en los que se sustentan los cambios estructurales planteados desde
\end{abstract}

\section{(1) $\Theta$}

Recibido: 19 de marzo de 2020. Aprobado: 2 de setiembre de 2020.

http://dx.doi.org/10.15359/rep.16-1.6

1 Académico. Bachiller en Enseñanza del Inglés (UNA). Master en Gestión Educativa con Énfasis en Liderazgo (UNA). Participante en diferentes eventos académicos internacionales en Costa Rica, Panamá, Chile, Cuba, Argentina y México. Identificador ORCID ID: https://orcid.org/0000-0001-6298-4509 
nuevas políticas educativas. Se identifican, de esta forma, las contradicciones entre los discursos y los hechos.

Palabras clave: Educación, política educativa, modelo educativo, sistema educativo.

\begin{abstract}
In November $17^{\text {th }}, 2017$, the Ministry of Education of Costa Rica (MEP, for its acronym in Spanish) published a new policy in terms of education. It emphasizes on positioning the validation of humans as diverse and complex and the necessity of educational practices that project equality and inclusivity for all the students in the educational system. However, when analyzing critically the political discourse of the ministry of education and the reality that is faced in the classrooms, it is possible to discover the real effects of the policy, many of them covered in the shadows of MEP. A new policy does not mean a change in the paradigm of education. In this academic essay, different theoretical referents are used to analyze the real implications and contradictions of the new policy as well as the neoliberal influences in the changes undertaken in the institution.
\end{abstract}

Keywords: Education, educational policy, educative methodology, educational system

\title{
Introducción al enfoque de análisis
}

bordar el desarrollo de la más reciente política educativa del
MEP implica un esfuerzo por entender sus repercusiones
desde el ámbito ideológico hasta el político y el pedagógico. Una política educativa conlleva esfuerzos que alcanzan el sistema en todas sus dimensiones, en la búsqueda de la calidad educativa, entendida desde la lógica de la institucionalidad, es decir, del Estado. Está claro que, en la gran mayoría de casos, el concepto de calidad parte de un entendimiento acorde a su funcionalidad en el sistema capitalista, enfocado en el cumplimiento y desarrollo de la educación según las perspectivas mercantiles e industriales, las cuales enfatizan la productividad y la competitividad (Muñoz, 2007). Sin embargo, para 
los efectos de este ensayo, es de suma importancia entender la calidad desde el planteamiento de Muñoz (2007): para que un sistema educativo cuente con calidad "es necesario que los procesos de enseñanza y aprendizaje dejen de estar bajo el control exclusivo del docente y pase a ser el resultado de una acción cooperativa entre el docente, los alumnos y la familia" (p. 175). Al entender la calidad educativa desde un paradigma constructivo que trasciende incluso la idea de los procesos pedagógicos encerrados en la institucionalidad, es posible analizar las repercusiones de las políticas impulsadas dentro del sistema educativo tanto en su contexto interno como el externo.

El planteamiento académico aquí realizado se fundamenta desde un enfoque metodológico permeado por el marxismo como un método de análisis de la realidad educativa. Esto implica comprender que la socialización de un sistema educativo corresponde a un proceso de reproducción del modelo económico y cultural, donde existen vínculos entre las practicas sociales y las valorizaciones culturales en función de mantener la hegemonía de la estructura de poder dominante (Giroux, 2004). Para lograr profundizar en este análisis se requiere comprender una política educativa y sus impactos en el sistema, a partir de las relaciones que se visualizan con las estructuras político-económicas y que se hacen presentes en el ejercicio hegemónico del poder dominante y su influencia en el sistema educativo.

\section{Política educativa de la persona: Centro del proceso educativo y sujeto transformador de la sociedad}

Durante 2017, el Consejo Superior de Educación promovió la más reciente política pública para situar la educación costarricense en una nueva etapa. La aprobación de esta reciente orientación política responde a dos factores fundamentales: por un lado, una nueva realidad social y, ante esto, una visión educativa emergente y; por otro, la variación de programas y reglamentos dentro del sistema educativo costarricense en los últimos años. Este cambio en la visión del modelo educativo ocurre después de 23 años de vigencia de la última política educativa, Hacia el Siglo XXI, aprobada por el Consejo Superior de Educación el 8 de noviembre de 1994. A partir de este planteamiento, surge la política educativa La persona: Centro del proceso educativo y sujeto transformador de la sociedad, cuya base elemental para 
fundamentarla corresponde al documento El centro educativo de calidad como eje de la educación costarricense, aprobado durante el 2008 (MEP, 2017). Desde esta aprobación es posible rescatar algunos de los elementos que podrían deslumbrar los orígenes y posibles repercusiones de este cambio.

A partir de su marco filosófico, se centra en diferentes paradigmas y corrientes del pensamiento científico, inicia por el paradigma de la complejidad donde se plantea un proceso educativo centrado en la persona como eje elemental de la enseñanza y el aprendizaje dentro del sistema educativo costarricense. De esta forma, esta política establece que "la educación, en consecuencia, está centrada en la persona, de manera que sea ella misma evaluadora y guía de su propia experiencia, a través del significado que adquiere su proceso de aprendizaje" (MEP, 2017, p. 9). La persona estudiante es actora activa dentro de su experiencia educativa y el bien primordial es alcanzar su crecimiento. Toma, además, elementos del constructivismo social como una herramienta para entender que las dinámicas educativas se encuentran vinculadas a un contexto social, a un entorno, en donde el centro educativo tiene un diálogo con los diferentes actores que interactúan con la institución. Sobre este marco, se menciona que "estos procesos se dan en asocio con comunidades de aprendizaje, dado que el conocimiento es también una experiencia compartida" (MEP, 2017, p. 10). Finalmente, retoma el paradigma del racionalismo como método científico que busca la objetividad como elemento central de los procesos. En esta línea, establece que "se sustenta en la razón y en las verdades objetivas como principios para el desarrollo del conocimiento válido" (MEP, 2017, p. 10). Conjugando estos diferentes planteamientos filosóficos, se orienta la base discursiva y reglamentaria de la política.

Partiendo de este enfoque filosófico, existen una serie de ejes programáticos en los que se sustenta la política. Estos mismos toman como punto de partida la calidad como eje central del resto de valores que la componen. El MEP (2017) define el rol de calidad "como principio nuclear que articula otros principios clave como la inclusión y equidad, el respeto a la diversidad, la multiculturalidad y pluriculturalidad, la igualdad de género, la sostenibilidad, la resiliencia y la solidaridad" (p. 10). Desde este punto de partida, se impulsan los ejes, iniciando por la $e d u$ cación centrada en la persona estudiante, donde se establece que todas las diferentes acciones que se tomen desde el sistema educativo deben 
impulsar su desarrollo en toda su integralidad y que el sistema debe funcionar en pro de la satisfacción de sus necesidades. Un segundo eje plantea la educación basada en los derechos humanos y los deberes ciudadanos, cuya meta es que las personas estudiantes tengan la capacidad de asumir estos derechos para alcanzar un mayor cumplimiento y efectividad tanto dentro del ministerio como de la sociedad costarricense, y generar, de ser necesario, cambios en el sistema en función de alcanzar el cumplimiento de estos derechos (MEP, 2017).

Un tercer eje se centra en la educación para el desarrollo sostenible, cuyo objetivo es que dentro de este proceso los sujetos se comprometan a construir sociedades basadas en una integralidad ambiental donde el ecologismo y la protección por la naturaleza representen elementos centrales (MEP, 2017). El eje siguiente, la ciudadanía planetaria con identidad nacional es descrito como "la toma de conciencia de la conexión e interacción inmediata que existe entre personas y ambientes en todo el mundo y la incidencia de las acciones locales en el ámbito global y viceversa" (MEP, 2017, p. 11). Este mismo plantea esa vinculación de la persona con un contexto globalizado. El quinto eje lleva el nombre de la ciudadanía digital con equidad social, desde donde se describe el conjunto de prácticas que busca reducir la brecha social existente por medio de la tecnología, se valora que existe un contexto donde a partir de condiciones sociales hay personas que no cuentan con el acceso suficiente a diferentes servicios básicos. Finalmente, se plantea un último eje encargado de la evaluación transformadora para la toma de decisiones que consiste "en la revisión continua con el fin de identificar los aspectos por mejorar, reconocer y hacer frente de manera positiva a la complejidad de los retos de la contemporaneidad" (MEP, 2017 p. 12). Desde este último eje, se visualiza el sistema educativo como uno en constante cambio y transformación. De esta forma, los ejes mencionados comprenden la propuesta educativa contemplada en la política.

La nueva política educativa del Ministerio de Educación Pública de Costa Rica impulsa una serie de orientaciones con el fin de alcanzar lo que ha titulado "una nueva realidad". De esta forma, plantea cuatro diferentes orientaciones: la transformación curricular, y de los espacios para la participación y la convivencia en el centro educativo; la transformación profesional docente: papel del personal docente y su formación permanente; la transformación institucional, y la transformación para 
una gestión educativa que redefina su nexo con el entorno (MEP, 2017). Cada una con una serie de elementos a considerar.

\section{Sobre la transformación curricular y de los espacios para la parti- cipación y la convivencia en el centro educativo}

En esta dimensión, el Ministerio considera diferentes señalamientos. Entre estos se encuentran una educación centrada en la persona estudiante y su proceso de aprendizaje, la educación como un proceso a lo largo de la vida y una ciudadanía del siglo XXI. Para alcanzar esta ciudadanía más acorde con las realidades emergentes, se establece la necesidad de trabajar en nuevas formas de pensar, de vivir el mundo, de relacionarse con otros seres humanos y obtener herramientas más acordes para integrarse a esta nueva realidad. Concreta además la necesidad de una ciudadanía digital e innovación y espacios para el vínculo, la convivencia y el desarrollo del potencial de cada persona en el centro educativo (MEP, 2017).

Establece, también dentro de sus orientaciones, el desarrollo progresivo de habilidades y competencias del siglo XXI, las cuales contemplan la educación de la primera infancia, la educación primaria, la educación secundaria, la educación técnica, la educación para personas jóvenes y adultas y la educación superior, cada una con una serie de compromisos y medidas. Sobre la educación de la primera infancia, se plantean dos grandes retos: la universalización de la educación preescolar y la articulación institucional para el avance y la optimización de recursos. Dentro de la educación primaria, nuevamente se retoma el compromiso con la universalidad de esta responsabilidad del Estado, el acceso a un currículo completo y el apoyo a los centros educativos con mayor rezago y vulnerabilidad. Desde la educación secundaria, la búsqueda de estrategias para la reinserción y la permanencia de estudiantes, mediante el apoyo personalizado, y la consolidación de un sistema con una propuesta diversificada, innovadora y de calidad debe entenderse como una necesidad prioritaria. En la educación técnica, el compromiso con el desarrollo de habilidades que faculten a la persona estudiante para la solución de problemas, la búsqueda de una movilidad social ascendente y el fortalecimiento de estrategias de formación que faciliten su inserción en el mundo del trabajo conforman el propósito elemental de este tipo de educación (MEP, 2017). 
A la educación para personas jóvenes y adultas también se le otorgan medidas, que inician por la búsqueda de una flexibilidad en los procesos de mediación y evaluación, y reconocimiento de aprendizajes construidos con la posibilidad de su certificación. Finalmente, la educación superior es dividida en educación parauniversitaria y universitaria. Sobre la primera, se menciona la posibilidad de generar mecanismos de vinculación con el mundo laboral. En cuanto al nivel universitario, se referencia al trabajo conjunto con las instituciones de formación de profesionales en educación y la importancia de la acreditación de carreras en esta área de estudio (MEP, 2017).

La última dimensión de trabajo planteada es la evaluación de los procesos educativos, desde donde se consideran diferentes elementos como la autoevaluación y construcción integrada, además de otras alternativas de evaluación, por ejemplo, establece que "los procesos evaluativos impulsarán la institucionalización de un programa nacional de uso de resultados basados en evidencias" (MEP, 2017, p. 19). Esto implica una serie de nuevas formas de comprender la evaluación dentro del sistema educativo.

\section{Sobre la transformación profesional docente: Papel docente y for- mación permanente}

Esta orientación va dirigida al personal docente en cuanto a su desempeño y formación. Desde ahí, se inicia planteando a la persona docente como alguien primordial en los procesos de aprendizaje. Se le adjudica el calificativo de "factor decisivo" para la formación de sus estudiantes, quienes a futuro serán la ciudadanía que tendrá que asumir retos y contribuir a su contexto social, a un desarrollo sostenible y a la responsabilidad social (MEP, 2017). Se hace referencia, además, al refuerzo de los valores, al énfasis en el uso de las tecnologías y al fomento del desarrollo intelectual como elementos integrales de las personas. Establece que "la persona docente abordará las problemáticas sociales y ambientales locales, regionales, nacionales y globales, de manera innovadora, crítica y reflexiva, con el propósito de formar en la persona estudiante, capacidades y responsabilidades ciudadanas" (MEP, 2017, p. 19). Estos aspectos son impulsados dentro del marco de las transformaciones propuestas por el Ministerio.

En cuanto a la formación inicial, se plantea un diálogo abierto entre el MEP e instituciones que formen profesionales en educación. 
Se recalca la importancia de que las personas que ingresen a carreras integradas a la docencia cuenten con habilidades de comunicación, aptitudes y perfiles éticos que favorezcan la docencia (MEP, 2017). Lograr este objetivo representa un reto, en especial para las universidades, en aspectos de formación y selección de quienes ingresen a sus programas.

Sobre el desarrollo continuo de la profesión docente, se plantean nuevamente una serie de retos. Se sustenta el trabajo por medio de redes de apoyo que busquen la atención de sus necesidades y también sus expectativas de mejorar en diferentes áreas de su labor profesional. Estas redes de apoyo pueden contar con la colaboración de instituciones que aborden, al igual que el MEP, la educación y su fortalecimiento. Es decir, tanto el MEP como otras instituciones educativas pueden abordar procesos de educación continua para profesionales de la educación. Un reto significativo se encuentra en el ámbito de la autoevaluación, vista como una medida para alcanzar el mejoramiento continuo de la labor docente (MEP, 2017). El sistema de autoevaluación se desprende como una medida para lograr identificar posibles espacios que impliquen transformaciones desde la labor cotidiana de la docencia.

El desarrollo continuo se encuentra, además, ligado a lo que el sistema educativo costarricense ha llamado el modelo de formación permanente. Desde ahí, se concibe al personal docente como agente de cambio en su entorno educativo. Por lo tanto, desde la formación permanente se busca reforzar esta orientación. Algunos elementos adicionales se encuentran en el liderazgo educativo, la contribución entre centros educativos y la construcción de redes de profesionales que se sustenten en procesos colaborativos (MEP, 2017). Estos procesos quedan abiertos a una implementación que puede ser tanto presencial como virtual.

\section{Sobre la transformación institucional}

Inicia con un planteamiento sobre la participación estudiantil, dándole un rol más activo en el proceso de aprendizaje. Desde este enfoque, se describe una participación estudiantil en la que incluso se presenta la posibilidad de que sea parte de la toma de decisiones en materia educativa. También se menciona una nueva vinculación con las familias para el apoyo en términos de permanencia y valor de los procesos educativos desde los hogares. En cuanto a la labor docente desde la mirada institucional, es descrito un esfuerzo para darle contundencia 
a procesos donde se pueda lograr que las personas que asumen la docencia sean quienes posean la mayor calidad; se enmarca, desde ahí, un sistema de méritos para incentivar el buen desempeño, generar coordinaciones, impulsar mecanismos para elegir las mejores candidaturas a los puestos de docencia y brindar asesoría a quienes se encuentran en su labor. Además, se proclama a la institución educativa, en conjunto con las comunidades, como el centro que va a nutrir las políticas educativas (MEP, 2017). También estás transformaciones implican aspectos del área de la infraestructura educativa, la superación de la barrera de la desigualdad, las direcciones regionales educativas y una cultura de rendición de cuentas, entre otras.

\section{Sobre la transformación para una gestión educativa que redefina su nexo con el entorno}

Uno de los aspectos que representa mayor desarrollo en la política se encuentra en la vinculación del sistema educativo con su entorno. Parte de la generación de redes entre los diferentes centros educativos del país que impliquen el impulso del trabajo colaborativo, indica, desde el concepto de comunidad educativa, la posibilidad de que sea un espacio abierto y con dinamismo que permita la contextualización de los procesos educativos para que incidan de forma pertinente en su realidad. Recalca la importancia de la participación de diferentes actores sociales y del liderazgo local dentro de las dinámicas institucionales. Para alcanzar esto, es importante la generación de alianzas con autoridades locales y comunitarias que puedan impulsar, incluso, programas culturales en conjunto. Finalmente, dentro de esta transformación, se mencionan otros cambios de importancia en cuanto a la vinculación con el entorno internacional, la articulación de la política educativa con otras políticas públicas y asumir la educación como una responsabilidad que le corresponde a todos y todas (MEP, 2017).

\section{Del discurso a la realidad: Análisis de las contradicciones}

Desde lo planteado en la política educativa antes descrita, es posible generar un análisis sobre los diferentes lineamientos y orientaciones para comprender sus impactos hasta la actualidad (año 2020), tres años después de su aprobación y divulgación. Asimismo, se vuelve necesario visualizar dónde se han dado las mayores transformaciones y qué implicaciones tienen, al igual que identificar dónde no ha existido cambio 
alguno con respecto a la realidad previa a esta política educativa para comprender las implicaciones del sistema educativo costarricense. Incluso, existe la posibilidad de encontrar contradicciones reales entre lo establecido en su aprobación y lo que se está realizando desde las aulas en las instituciones; contempla el verdadero rostro de los procesos, ese que se encuentra en la acción diaria dentro de los centros educativos. Esto, debido a que las clases son el escenario donde se deben experimentar las mayores transformaciones en función de las personas y el proceso educativo. Para abordar este análisis, se contemplan diferentes elementos que han implicado debate y discusión en diversos espacios académicos, se contrastan realidades teóricas con las orientaciones en la Política educativa de la persona: Centro del proceso educativo y sujeto transformador de la sociedad. Al partir del entendimiento de que la educación es una responsabilidad del Estado en la búsqueda de la conformación de una nueva ciudadanía, se vuelve de gran significado la reivindicación de los procesos educativos en la construcción de esa identidad ciudadana. Como lo plantea Gramsci (2014), "en realidad el Estado debe concebirse como educador, en cuanto que tiende, precisamente, a crear un nuevo tipo o nivel de civilización" (p. 400). Desde este enunciado, este ensayo académico realiza un análisis crítico de la realidad educativa en Costa Rica.

\section{Influencia empresarial: Educación dual}

Dentro de las orientaciones de la política educativa, es posible observar una serie de elementos que demuestran la vinculación con los requerimientos empresariales del momento. Partiendo de aquí, es necesario recalcar que existe un debate histórico entre los diferentes enfoques que podría tener un modelo educativo. Por un lado, un enfoque donde se posiciona al sistema educativo en función de las exigencias del mercado. Por el otro, un proceso donde desde el sistema educativo se busca contribuir en las necesidades de las personas y las comunidades. Ambos enfoques rozan uno con el otro en sus principios, fines y elementos. En cuanto a la política educativa, desde el MEP, al ser una institución al servicio del Gobierno de turno, ha sido posible palpar una orientación constante hacia prácticas cada vez más cercanas a los requerimientos neoliberales, principalmente en los últimos 40 años. Es decir, surgen diferentes iniciativas políticas e ideológicas con el fin de colocar el Estado en función de las exigencias del poder económico, las 
élites burguesas y la clase empresarial donde muchos de estos cambios llegan disfrazados en el discurso de la inserción de las personas estudiantes en el mundo laboral. En esta línea, la política educativa plantea que "se fortalecerán estrategias de formación profesional y educativa que garanticen la inserción de las personas jóvenes al mundo del trabajo, donde la educación dual representa un ejemplo de ello" (MEP, 2017, p. 18). La discusión más reciente en la cual se debaten estas visiones o enfoques de los sistemas educativos se observa en el planteamiento de procesos de educación dual.

La educación dual corresponde a un modelo importado desde corrientes europeas, representa un proceso que demuestra que aún persiste la influencia colonialista en los sistemas educativos, pues se repeten sus recetas en países latinoamericanos. Esta modalidad de la educación busca que las empresas puedan obtener el servicio de estudiantes practicantes y, a cambio de este, los grupos estudiantiles puedan obtener experiencia laboral. En un principio, si prevalecen procesos educativos de calidad y las condiciones laborales se desenvuelven dentro de un marco de reconocimiento de derechos al igual que el de las personas trabajadoras, no tendría por qué satanizarse este modelo. Sin embargo, ese no es siempre el escenario. "Con este modelo las empresas aprovechan las pasantías de los estudiantes a costos mínimos, dejando de lado las regulaciones y las posibles intervenciones estatales que busquen garantizar una educación de calidad" (Robles, 2017, p. 28). Justamente esta descripción, en la cual no prevalece un principio de calidad en la educación, se puede visualizar en la ley aprobada para la regulación de la educación dual en Costa Rica.

Durante el año 2019, la Asamblea Legislativa de Costa Rica aprobó un proyecto para la implementación de la educación dual en el país. Este, a pesar de que en el discurso político se buscó hacer referencia a una influencia del modelo alemán, contempló una serie de contradicciones en cuanto a lo que por el fondo planteó la iniciativa. En Alemania, la educación dual implica que las personas estudiantes que ingresan a este tipo de modelo educativo requieran de un proceso previo de análisis, realizado por una comisión tripartita conformada por representantes del ministerio educativo, empresariado y sector trabajador. Tal y como indican Rindfleisch y Maenning-Fortmann (2019), sobre el caso alemán, "la Ley de formación técnica establece que los empleadores, los trabajadores y el estado son los encargados de fijar en 
conjunto las condiciones marco para la formación dual" (p. 23). Una vez concretado un acuerdo sobre las condiciones del procedimiento a seguir, se recibe la persona estudiante en la empresa. Esta labor implica un contrato, salario e incluso seguro social.

En cuanto al proyecto costarricense, la realidad es otra. El proyecto en la Asamblea Legislativa inicialmente reconocía el pago de horas al estudiando en este tipo de formación. A partir de una recomendación de la Unión de Cámaras Costarricenses y Asociaciones del Sector Empresarial Privado (UCCAEP), por medio de una carta, las diputaciones en la comisión que estudiaba el proyecto cambiaron la obligatoriedad en el pago de este reconocimiento por la labor brindada, dejando a la libre, si la empresa de buena voluntad lo hacía o no. Al estudiar la Ley de Educación y Formación Técnica Dual (2019) es posible concluir que el proyecto costarricense no fue uno igual a los impulsados en Europa, copiado de la "exitosa" práctica alemana. En el fondo, es un proyecto característico de un país donde las cámaras empresariales dictan el rumbo de la clase política para obtener réditos, en este caso, posible mano de obra barata. Uno de los elementos más interesantes que eliminaron en el texto es la figura de la relación laboral entre estudiantes en formación dual y la empresa. Esto se puede constatar en el artículo 22 inciso i) donde se plantea: "Considérese aprendiz al estudiante únicamente para la aplicación del Título IV del Código de Trabajo" (Asamblea Legislativa, 2019, p. 17). Planteando este análisis de manera figurativa: si tiene plumas, pone huevos y suena como gallina, pues entonces es una gallina. No un gato, ni un perro. Desde ahí, si una persona trabaja, brinda un servicio, cumple un horario y tiene un jefe. ¿Qué es? ¿Una persona trabajadora o no?

Al tomar como referencia el valor de la plusvalía desde el marxismo, es evidente que la ausencia de esta relación obrero-patronal, en la educación dual, trae consigo que a las personas estudiantes no se les reconozca su fuerza de trabajo, mientras que la persona empresaria tendrá una mayor ganancia. Este fenómeno consiste en que la fuerza de trabajo de la persona obrera produzca un valor mayor del que se ha contemplado en su salario, por lo tanto, ese valor mayor no reconocido es la plusvalía (Arnaudo, 2013), realidad que ya existe en las relaciones laborales convencionales y bajo el marco jurídico del trabajo formal, pero que, en condiciones como la educación dual, simplemente se amplía al contar con menos derechos laborales. 
Al analizar este ejemplo particular, es posible observar las interacciones que se dan desde las cámaras empresariales en la generación de políticas educativas. El mismo MEP (2017) indica que "ha facilitado la implementación de la educación dual" (p. 7). Además, la institución no planteó mayor objeción al proyecto aprobado en la Asamblea Legislativa, lo cual dejó en evidencia que muchos de los cambios en el sistema educativo no van en función de las personas, sino más bien de las empresas, es decir, el capital, que solicitan ciertos estándares o lineamientos para obtener un mayor aprovechamiento de sus recursos y generar réditos económicos.

\section{El discurso sobre el pensamiento crítico}

En este ámbito, es posible identificar nuevamente un debate en cuanto a la interpretación del pensamiento crítico como concepto epistemológico. Primeramente, sería oportuno definirle como "una forma de adquisición de conocimiento, no la única, pero si la más valiosa, al menos para el ser humano" (Saiz, 2019, p. 19). Sobre este concepto, ha crecido una tendencia desde el poder económico por el desarrollo de este tipo de razonamiento, siempre que este mismo esté abocado a la resolución de conflictos, toma de decisiones y pensamiento creativo. Es decir, un enfoque constructivo, pero que no atente contra el status $q u o$ o el poder político-económico hegemónico. Esta tendencia busca la incorporación de personas críticas dentro de la fuerza laboral que las empresas requieren y habla, incluso, de que este tipo de habilidades trascienden la formación profesional. La era en la que no importa qué título se posea, sino qué habilidades puedan tener. Diferentes ramas de la psicología y la filosofía han contribuido en este proceso de construcción del concepto, definiendo, entonces, el pensamiento crítico como "alcanzar la mejor explicación para un hecho, fenómeno o problema con el fin de saber resolverlo eficazmente" (Saiz, 2017, según se cita en Saiz, 2019, p. 25). Se palpa en esta definición conceptual, el entendimiento del pensamiento crítico como un proceso de razonamiento que no tendría por qué incidir en el ámbito político y, por tanto, tampoco en el económico.

Por otro lado, desde un enfoque latinoamericano y, particularmente desde la pedagogía, también existen aportes sobre el pensamiento crítico, pero esta vez desde un abordaje que implica el cuestionamiento de estructuras y la desconstrucción de imposiciones sistemáticas. Desde 
esta percepción pedagógica, el pensamiento crítico se puede concebir como "un acto de conocimiento y un proceso de acción transformadora que debería ejercerse sobre la realidad" (Freire, 1985, p. 116). Aquí existe una diferencia elemental sobre qué se entiende por pensamiento crítico en otras corrientes de análisis, en este caso, se asume que la persona tiene la posibilidad de incidir en su realidad social, política y económica e incluso generar transformaciones. Evidentemente, esta posición atenta directamente con el status quo y las estructuras sociales que trabajan en función de ciertos sectores. Se vuelve fundamental entender que "una cosa es la relación entre teoría y la práctica en una educación orientada hacia la liberación, y otra muy distinta es una educación cuyo objetivo es la 'domesticación'” (Freire, 1985, pp. 37-38). A partir de esta diferenciación entre lo que se puede entender como pensamiento crítico y a qué fines responde, es posible analizar la política educativa del MEP en Costa Rica.

Desde esta política, se establece que el estudiantado tiene un papel fundamental en el impulso de un pensamiento crítico, indica que las personas estudiantes "sean informadas, consultadas e involucradas en la toma de decisiones y el acompañamiento de los temas que les competen" (MEP, 2017, p. 21). Sin embargo, es posible identificar que el pensamiento crítico orientado en las políticas educativas responde nuevamente a un enfoque que se enmarca dentro de los intereses empresariales, procesos que no atentan, y mucho menos cuestionan, las estructuras sociales preestablecidas. Es posible denotar el esfuerzo por alcanzar un pensamiento crítico que no emancipe, ni mucho menos libere, sino que esté en función de brindar elementos que luego puedan ser explotados según los requerimientos del mercado. En este sentido, es fundamental comprender, como lo plantea Freire (1985), que "sería extremadamente ingenuo pretender que las clases dominantes desarrollen un tipo de educación que permitiera a las clases sometidas percibir críticamente las injusticias sociales" (p. 115). Quedan nuevamente en evidencia las orientaciones que persigue el modelo educativo costarricense, incluso cuando retoma conceptos como pensamiento crítico u otros de suma importancia en materia pedagógica.

\section{Vinculación con la comunidad y realidad social}

Uno de los elementos que tienen mayor atención dentro de la política es la vinculación del sistema educativo con la comunidad y 
su realidad. Desde esta perspectiva, es importante resaltar la influencia que existe en un centro educativo con respecto a la construcción de una identidad cultural y lo necesario de un proceso de contextualización de todo lo que corresponda a un currículo educativo, teniendo en cuenta que la imposición de un conocimiento ajeno a la realidad del estudiantado podría no tener los mejores resultados en los procesos de aprendizaje y enseñanza al pertenecer a una realidad diferente y lejana a su contexto. Dicho de otra manera, el conocimiento que no tiene incidencia en el contexto donde se encuentra la institución, termina con muy poco impacto en su formación. Sobre esta práctica explican Freire y Shor (2014) que "el conocimiento les es dado como un cadáver de información - un cuerpo muerto de conocimiento- y no como una conexión viva con la realidad de cada uno de ellos" (p. 21). Para combatir esto, el MEP (2017) menciona en la política educativa que:

Cada centro educativo desarrollará su potencial y particularidades, de acuerdo con su contexto, atendiendo a sus especificidades regionales y territoriales. Lo anterior permitirá la consolidación de un proyecto curricular de centro, proceso en el cual el liderazgo del director o directora será fundamental. (p.15)

Esto implica un proceso educativo que es abordado desde una vinculación y adaptación directa a su contexto de manera que el trabajo curricular que se desarrolla en un centro educativo sea un proyecto que trasciende a lo comunitario para lograr contribuir e incidir desde la institución en su realidad inmediata. Esto encuentra sentido incluso desde enfoques latinoamericanos sobre la pedagogía de la liberación. Desde esa perspectiva, Freire (1987) menciona que "por ser educación, habría de ser valiente, ofreciendo al pueblo la reflexión sobre sí mismo, sobre su tiempo, sobre sus responsabilidades, sobre su papel en la nueva cultura de la época" (p. 51). En otras palabras, se posiciona a la educación en función del pueblo y la transformación de su realidad social, sus problemáticas e intereses.

El sistema educativo en esta política remarca aspectos como el servicio comunitario que deben realizar las personas estudiantes para establecer canales de comunicación con diversos sectores sociales. Describe el MEP (2017): 
El sistema educativo potenciará el desarrollo del servicio comunal estudiantil en distintas etapas de la formación educativa, para promover la interacción de los talentos y las habilidades estudiantiles con las necesidades sociales de su contexto. Este servicio será parte esencial de la formación de la persona estudiante con respecto a sus deberes ciudadanos. (p. 15)

Sin embargo, al analizar el desarrollo de estas actividades dentro de los centros educativos, es posible observar poca vinculación o avance en términos de servicios comunales, no solo por parte de estudiantes, sino que además docentes, e incluso las instituciones, como un todo, no abordan labores que verdaderamente contemplen los criterios de las comunidades y las exigencias de sus realidades, mucho menos alcanzar un intercambio de saberes con actores comunitarios. En este sentido, la mayoría de aportes se basan en acciones que van más en la línea de actuaciones voluntariosas de las personas estudiantes o los centros educativos, pero no existen adaptaciones de contenidos, ni contribución del contexto en los abordajes metodológicos, es decir, la contextualización no llega al proceso didáctico y mucho menos al pedagógico.

En muchas ocasiones, el centro educativo pierde toda comunicación con la comunidad o incluso se vuelve un diálogo unidireccional en el que la institución es la que domina el discurso y lo forja de acuerdo con los intereses del sistema educativo. La vinculación con la comunidad queda constituida en las figuras políticas de carácter administrativo con la formalización legal de las juntas de educación, las cuales tienen la responsabilidad de coordinar acciones de carácter administrativo en el centro educativo. Mientras que, en lo curricular, no existe influencia de la comunidad sobre los contenidos que se abordan desde el centro educativo. En esta realidad, las direcciones de las instituciones incluso pierden legitimidad ante la comunidad, a pesar de que el MEP (2017) describe el rol de los directores y directoras de centros educativos como "autoridades educativas locales" (p. 23). Es decir, existe una desconexión entre la institución y la comunidad. Aquí se puede retomar una contradicción de carácter político-ideológico, es posible identificar una conexión entre altas esferas y entes tomadores de decisiones en materia educativa y sectores del poder económico y empresarial. En esta línea, nuevamente el MEP (2017) indica que "la apertura de nuevos espacios de diálogo e intercambio con las organizaciones laborales y 
las empresariales contribuirá a alimentar al sistema educativo con información clave relativa a áreas emergentes y nuevas necesidades del desarrollo social y productivo" (p. 26). Como se ha mencionado anteriormente, este vínculo ha encaminado al modelo educativo a un proceso en función de los intereses del mercado, más que de las personas y las comunidades, y sus necesidades, intereses que si se pueden ver plasmados en una contextualización de políticas, currículos, metodologías y procesos educativos.

\section{Rol docente: Implicaciones en el desempeño}

La persona docente juega un papel primordial dentro del sistema educativo. Diferentes estudios abordan la complejidad de sus funciones y la influencia que posee en los resultados de un proceso educativo. Uno de los aspectos de debate constante es la intelectualidad que debe tener la persona docente para el ejercicio de su profesión. En esta línea, Gramsci (2014) plantea dos tipos diferentes de intelectuales, uno de corte tradicional y otro orgánico, describe:

Orgánicamente los intelectuales de aquellas masas, lo que quiere decir: esa organicidad solo podría conseguirse si los intelectuales hubieran elaborado y hecho coherente los principios y los problemas que planteaban aquellas masas con su actividad práctica, constituyendo así, entre unos y otros un bloque cultural y social. (p. 370)

Basado en esta clasificación, Gómez (1987) define la función de estos intelectuales como un proceso donde "se ligará orgánicamente a la masa a través de una pedagógica actividad en la que aparecerá una íntima relación entre conocer y querer, consciencia del fin y programa de acción, autodisciplina y ejecución, teoría y práctica" (p. 72). Desde la docencia, este rol implica una labor pedagógica que aporta desde la intelectualidad para que, en conjunto con las masas, se logre encontrar solución a sus necesidades y más prontas demandas. Esas masas se encuentran representadas por la población estudiantil y las comunidades donde se encuentran los centros educativos.

Partiendo de esta percepción teórica, el MEP establece, desde su política orientadora de la educación, la idea de un personal docente descrito como un intelectual orgánico. A pesar de que no se utiliza 
directamente el concepto, se describen algunas de sus funciones, que lo plantean como "agente de cambio en la comunidad educativa" (MEP, 2017, p. 21). Esto implica que la persona docente debe tener las capacidades de interactuar en la realidad social del contexto en el que se desenvuelve, aportar desde sus saberes y contemplar, además, los conocimientos de las comunidades. Sin embargo, al realizar un balance con la realidad, es posible identificar patrones contrarios a la descripción impulsada desde la política educativa en las aulas de los centros educativos. En gran medida, a la persona docente se le direcciona en un rol que se limita a la repetición de las directrices dadas por el currículo oficial en donde los planeamientos educativos se convierten en copiar y pegar lo establecido desde el Ministerio. Esto se puede constatar en los formatos y requisitos mínimos que se solicitan en los planeamientos de las diversas materias educativas. Desde esa realidad práctica, la persona docente no se desenvuelve como alguien con una intelectualidad orgánica, tampoco como agente de cambio, sino que asume una posición de replicadora de conocimientos técnicos en favor de las estructuras establecidas.

En cuanto a los procesos de contratación docente, se mencionan la importancia de generar cambios en donde prevalezca la calidad, se indica que se van a "c) impulsar mecanismos para elegir a los mejores candidatos y candidatas a la docencia" (MEP, 2017, p. 22), además de indicar que "el sistema educativo atraerá a los mejores candidatos y candidatas a docentes" (p. 22). Por lo tanto, desde el MEP, se considera que mejorando el personal docente mejorará la calidad de la educación que se imparte. Sin embargo, los métodos de contratación no han variado. Se siguen dando procesos donde las personas son contratadas a partir de sus atestados y el cumplimiento de los requisitos para el puesto, lo cual se puede constatar en el más reciente proceso de reclutamiento docente, Concurso Docente 2019, donde para ser oferente y estar dentro del sistema educativo debieron nada más cumplir con los atestados solicitados. No existe, hasta el momento, ninguna entrevista previa ni vinculación con el contexto laboral.

\section{Rol de la persona estudiante: ¿Activa o pasiva?}

El rol de la persona estudiante tiene un papel sumamente activo en la realidad educativa. Sobre la activa participación y la inversión de roles dentro de los procesos de aprendizaje, Gramsci (1967) indica que 
"este problema puede y debe ser asemejado al moderno planteamiento de la doctrina y la práctica pedagógica, según el cual la relación entre maestro y alumno es activa (recíproca), donde el maestro sigue siendo alumno y el escolar, maestro" (p. 91). En ese sentido, el rol de una persona estudiante formada en un modelo crítico, trasciende la repetición de conocimiento o ejercicios evaluativos memorísticos. Implica, por el contrario, la toma de decisiones, la creatividad y la construcción de conocimientos, un proceso que además debe ser inclusivo y garantizar la participación de todas las personas. En este sentido, el MEP (2017) establece:

La mediación en el aula partirá del paradigma de la atención y el reconocimiento a la diversidad, y como condición imprescindible, garantizará la igualdad de oportunidades y el desarrollo de habilidades, según las capacidades, ritmos de aprendizaje y posibilidades de cada quien, al asumir la variabilidad como norma y no como excepción. (p. 16)

Este proceso representa la atención individualizada y acorde con las necesidades de cada persona, y asume que la educación debe estar permeada por un principio de inclusividad de forma que nadie se sienta relegado o que no forma parte del proceso de aprendizaje.

Uno de los mayores retos en esta línea es enfrentarse a viejos modelos de aprendizaje, paradigmas que no garantizan espacios donde estudiantes y docentes puedan ir más allá de sus dicotómicos roles institucionalizados. Freire (1985) hace mención a este fenómeno al comentar que "a los educandos no se les llama para que participen creativamente en el proceso de aprendizaje, sino que se les 'rellena' con las palabras de los educadores" (p. 115). Muchas veces estas palabras que se usan de relleno incluso no responden a las personas educadoras, sino a libros de texto replicados de forma incuestionable. A la vez, se puede identificar la medición del conocimiento enfatizada en evaluaciones que piden la repetición a ultranza de esa información y en donde, si no se mencionan conceptos exactos, las respuestas se toman como incorrectas. Dentro de estos procedimientos, se deja de lado el análisis fundamentado, la búsqueda de nuevas formas de entender las situaciones y la propuesta de diferentes maneras de entender el conocimiento. 
Este tipo de evaluación está estrechamente ligado a la formación de un pensamiento único en función de lo que se espera como correcto y acorde a intereses particulares. Estas características se pueden atribuir al concepto marxista de la masificación, el cual también fue desarrollado en el ámbito pedagógico por Paulo Freire. La masificación implica la homogenización del comportamiento de las masas en función de las estructuras de poder desde el espacio educativo (Freire, 1987). De esta manera, las personas, posteriormente, pueden incorporarse a las dinámicas sociales que contribuyen al sistema capitalista; por ejemplo, al contemplar las mismas formas de consumo, es posible conocer con mayor claridad el comportamiento de los mercados, además de facilitar procesos de dominación.

\section{Conclusiones generales}

Un análisis pausado de las implicaciones de las políticas educativas impulsadas desde el MEP debe tener la capacidad de visualizar y predecir sus repercusiones. A partir del estudio de esta política, es posible concluir que la mayoría de aportes que se dan desde esta nueva propuesta educativa recaen en aspectos generalmente ideológicos, fundamentados desde un enfoque teórico que no llega a impactar la realidad práctica. Los únicos escenarios diferentes son aquellos en los que existen fuertes intereses ideológicos, donde se perciben cambios más allá del aparato discursivo y encuentran modificaciones en torno a la visión pedagógica e, incluso, aspectos didácticos que se llevan a cabo dentro de las instituciones. Recapitulando: existen cuatro planteamientos fundamentales que podrían orientar la discusión sobre los alcances de esta política educativa y el porvenir:

1. Es fundamental identificar los alcances de la educación como un acto profundamente político, esto, más allá de que las personas docentes estén al tanto o no de su rol. Es decir, todo desempeño que se realiza dentro de un espacio educativo tiene una implicación política que responde a los intereses de un sector o actor social. Freire (1985) nos recuerda:

La educación, sea en la universidad, la escuela secundaria, la primaria o en la alfabetización de adultos, es un acto político. ¿Por qué? Porque la naturaleza misma de la educación tiene las cualidades inherentes para ser política, así como la política posee 
aspectos educativos. En otras palabras, un acto educativo tiene naturaleza política, y un acto político tiene naturaleza educativa. (pp. 184-185)

Esto significa que la puesta en marcha de una nueva política educativa refleja los intereses políticos de un grupo social por alcanzar un número de metas y expectativas de acuerdo a sus intereses, que se encuentran reflejados en la idea o conceptualización sobre la nueva ciudadanía que se formará con esta política educativa. Muchos de estos intereses se encuentran desde los discusivo y, es posible, percibir sus cimientos ideológicos en función de los intereses mercantiles más que sociales.

2. Es posible identificar una serie de contradicciones elementales entre lo que se plantea en la Política educativa de la persona: Centro del proceso educativo y sujeto transformador de la sociedad y la realidad práctica dentro del sistema educativo a tres años de su implementación. Muchas de estas contradicciones se vislumbran en un discurso educativo que no parece ser la verdadera orientación del sistema, nuevamente, estas contradicciones se sustentan principalmente en lo ideológico, pero se pueden palpar más en la realidad práctica desde los procesos pedagógicos.

3. Existe una tendencia evidente de un modelo educativo que se inclina hacia la formación técnica al servicio de las lógicas del mercado, lo cual se visualiza como la posibilidad de preparar personas con la capacidad de realizar funciones técnicas de calidad y que representen mano de obra barata en términos de contratación. En este camino, se deja cada vez más de lado, en la práctica, aunque no en el discurso, la implementación de un modelo educativo en búsqueda del crecimiento intelectual y, por tanto, de un pensamiento crítico transformador que implique la desconstrucción de realidades, atentar con normas establecidas que representen actos de injusticia y desigualdad, y cambios en las estructuras sociales imperantes. Nuevamente, esto representa una clara realidad política de los intereses a los que responde el modelo educativo.

4. La contextualización de un proceso educativo se asume desde la necesidad de que exista una vinculación con la comunidad 
y establecimiento de puentes diálogos y trabajo conjunto con diferentes actores sociales. En la práctica, este esfuerzo esta caracterizado por un avance lento a una realidad de intercambio de saberes que, al contrario, parece cada vez más lejano. Los roles tanto de estudiantes como docentes deben romper con las estructuras dicotómicas y los planteamientos unidireccionales, en los cuales la persona docente es dueña absoluta del proceso educativo. En este sentido, existe, a la vez, una relación de poder unidireccional entre el currículo educativo y la persona educadora, es decir, la persona docente se ve obligada a acatar y cumplir con lo planteado en el currículo, sin importar su significancia en el contexto. De esta forma, esa relación se replica luego en la mediación entre docente y estudiante, pero esta vez en los hombros de la persona docente, quien exige el cumplimiento de lo establecido en el currículo, sin miramientos. Además, la acción política del sistema educativo se cumple con el beneplácito de la persona educadora consciente o inconscientemente de su labor política. Aquí el rol del estudiantado se mantiene en una participación pasiva, aunque en la política se indique otra cosa, una contradicción de carácter pedagógico de grandes repercusiones.

Estos cuatro elementos deben contribuir a una discusión que logre trascender del espacio académico y alcanzar espacios populares, comunitarios y de intelectuales independientes, donde es fundamental debatir sobre las expectativas de lo que el modelo educativo costarricense debe o debería ser. Sobre este fenómeno, es necesario retomar marcos de referencia entorno a una nueva concepción de lo que se entiende por calidad educativa, y dejar de lado las fuertes influencias que existen en el posicionamiento de un concepto estático para alcanzar una idea de calidad más constructiva y dialéctica, un debate que pueda reflexionar sobre qué nuevos paradigmas evaluativos son necesarios para superar las influencias del positivismo. Se debe analizar la posibilidad de incorporar procesos extensionistas críticos desde niveles educativos inferiores a la educación universitaria que contribuyan a dar un paso al frente para salir de vínculos comunitarios sustentados en el voluntarismo o un activismo sin orientación. Finalmente, se vuelve relevante una reflexión sobre la importancia de reconocer otras formas de saberes que 
involucren los aportes desde ideas ancestrales para que logren contribuir al entendimiento de la realidad histórica y cultural que constituye la sociedad costarricense; que reconozcan el contexto y la circunstancias en las que viven las personas, sin necesidad de homogenizaciones ni imposiciones sistemáticas. Son estos y muchos otros temas los que requieren de un estudio y debate que logre contribuir en la generación de bibliografía alternativa en el ámbito educativo y la construcción de una comunidad de saberes que, a la larga, pueda generar incidencia y aportes importantes en su realidad inmediata.

\section{Referencias}

Asamblea Legislativa. (2019). Ley de educación y formación técnica dual. La Gaceta, San José, Costa Rica.

Arnaudo, F. (2013). Teoría de la plusvalía en Marx. Revista Cultura Económica, 31(86), 43-49. Recuperado de https://repositorio.uca. edu.ar/bitstream/123456789/2111/1/teoria-plusvalia-marx-arnaudo.pdf

Freire, P. (1985). La naturaleza política de la educación: Cultura, poder y liberación. Barcelona: Centro de Publicaciones del Ministerio de Educación y Ciencia y Ediciones Paidós Ibérica.

Freire, P. (1987). La educación como práctica de la libertad. México, D. F.: Andrómeda.

Freire, P., y Shor, I. (2014). Miedo y osadía. Buenos Aires: Siglo Veintiuno Editores.

Giroux, H. (2004). Teoría y resistencia en educación. México: Siglo XXI-UNAM.

Gómez, J. (1987). Intelectuales y pueblo un acercamiento a la luz de Antonio Gramsci. San José: Departamento Ecuménico de Investigaciones.

Gramsci, A. (2014). Antología Volumen 2. Buenos Aires: Siglo Veintiuno Editores.

Gramsci, A. (1967). La formación de los intelectuales. México: Editorial Grijalbo.

MEP. (2017). Política educativa de la persona: Centro del proceso educativo y sujeto transformador de la sociedad. San José, Costa Rica: Autor. 
Muñoz, C. (2007). Un nuevo paradigma: "La quinta generación de la evaluación". Lauros, 13(23),158-198. Recuperado de http:// www.redalyc.org/articulo.oa?id $=76102309$

Rindfleisch, E. y Maenning-Fortmann, F. (2019). Formación dual en Alemania formar técnicos por medio de la teoría y la práctica. Alemania: Konrad Adenauer Stiftung.

Robles, A. (2017). Sistema educativo costarricense: ¿Puerta o barrera para el pensamiento crítico? Revista Ensayos Pedagógicos, 12(2), 17-31. https://doi.org/10.15359/rep.12-2.1

Saiz, C. (2019). Pensamiento crítico y eficacia. Madrid: Ediciones Pirámide. 